\title{
Quality Improvement Teams: Moving from the Passionate Few to the Mandated Many
}

\author{
Ann Lefebvre, MSW, CPHQ
}

Quality Improvement has gone from practice by practice piecework to an industry driven by expertise that lies within corporate offices rather than within patient care. Using for her 20 years of experience leading and teaching quality improvement as a lens, the author makes the case for quality improvement teams to ensure a key role for clinicians and direct care staff who are closest to the patients and the improvements that need to be made. (J Am Board Fam Med 2020;33:S42-S45.)

Keywords: Certification, Data Accuracy, Physicians, Quality Improvement

As we celebrate 50 years of the specialty of family medicine and we look at how the practice of medicine has changed, we can see the advent of quality improvement (QI) and its impact in our work as we weigh the value of the specialty and our ultimate outcome in the health of the patients served. Throughout my career, I have had the opportunity to experience firsthand the development of the field of QI from a movement of the willing to a required mandate within our health care system and the attempts made to measure that impact over time.

In the mid 1990s I experienced QI as an extracurricular activity, a specialized sport, that involved only those personnel who were interested enough to dabble in it during our "off hours." QI spoke to me in a way that many areas of my chosen field did not. As a social worker supporting patients with infectious diseases, I was a firsthand witness to poor patient outcomes and the numerous processes within our health care system that appeared counterintuitive to patients' needs. QI techniques provided me the ability to look at processes and the impact that a good process could have on a patient's experience within our system. It felt good when something worked well and I enjoyed being able

This article was externally peer reviewed.

Submitted 3 January 2020; revised 18 June 2020; accepted 22 June 2020.

From the South Carolina Area Health Education Consortium; Department of Family Medicine, Medical University of South Carolina, Charleston.

Funding: None.

Disclosures: None.

Corresponding author: Ann Lefebvre, MSW, CPHQ, Medical University of South Carolina, 1 South Park Circle, Suite 201, Charleston, SC 29407 (E-mail: lefebvre@musc. edu). to spread that to other systems to impact other people and their jobs and their experiences. It was a way to help people manage change in a positive manner. It gave a voice to all staff in our practice and it made sense to me.

As I progressed further in my career, I was able to apply these methods to hundreds of communitybased primary care practices that trusted me to help them redesign their care delivery systems and to implement registries and electronic health records in a way that supported their practices and their interest in improving their patients' health. ${ }^{1}$ By 2008 , I had developed a formalized practice coaching program; and by 2018 , we had deployed practice coaches to more than 1500 primary care practices across North Carolina and improved health outcomes in diabetes and heart disease. ${ }^{2,3}$ This work would allow many of these practices to achieve better contracts with payers or to successfully move into integrated health systems with sophisticated projective analytics, patient satisfaction ratings and value-based payment mechanisms. We had also laid foundations for other programs like it in many states across the country. ${ }^{4}$

Over this time, the field of QI was evolving as well. In 1997, Health Affairs published a commentary by Elizabeth McGlynn ${ }^{5}$ that listed 6 challenges in measuring quality in the US Health care system. The 6 challenges she outlined are:

1) Identify and balance the competing perspectives of the major participants in the health care delivery system, 2) develop an accountability framework, 3) establish the explicit criteria by which health system performance will be judged, 4) select a subset of indicators for routine reporting, 5) minimize the conflict 
between financial and nonfinancial incentives and quality-of-care objectives, and 6) facilitate the development of information systems necessary to support quality monitoring.

At the time that it was written, I was captivated by Dr. McGlynn's ideas and energized by her vision for where the field of QI could take our systems of care. Even now, after decades of working to help build a better approach at the local, state, and national levels, I am struck with how relevant the challenges identified in 1997 still are today. While we have made gains including the development of national quality metrics, payment toward those metrics and the capability of informatics systems to identify and gather data, we have contributed to or created further challenges such as different metrics being used by different payers for the same services or the divergence between clinical guidelines and some pay for performance measures.

What used to be called "cookbook medicine" is now recognized and accepted as evidence-based protocols and care pathways that provide a sophisticated and reliable means to ensure that patients get the right care at the right time in the right way. However, I worry that we may be moving to a time of “cookbook QI" where we no longer dig as deeply into patient outcomes to learn where to improve or perform root-cause analyses to more fully understand the data we have. We rely heavily on teams of analysts who work and rework the data to force it to show improvement. If we are not careful, improvement may present in the data with little impact on patient care or the patients' health. While improving our ability to collect and understand data are important, has it taken over the driver's seat? Are we simply working to improve the data?

It is important to recognize that while improving the ability to collect and analyze data are a part of QI, it is not the entirety of improvement. In fact, it is only the beginning. For example, I have worked with practices to improve the hypertension outcomes within their patient population. A typical place to start the project would be to retrain staff on appropriate ways to take a patient's blood pressure. The practice should develop protocols on when to retake blood pressure to get an accurate reading, especially if it was out of acceptable range. While both actions are a good place to start a hypertension QI project and both actions may improve the data and provide a more accurate reading for which the provider to use for their clinical decisions, neither of these undertakings will actually improve the patient's hypertension. The patient's blood pressure remains the same whether we measure it accurately or not. While still an important part of the process, those efforts only improve the data. Accurate data are necessary but can be a deceitful part of QI because it may look like an improvement in a health outcome when it is only an improvement in the data collection. We must ensure that we are pushing ourselves to go further and improve the health of the patient.

McGlynn addresses this in her challenges and mentioned the importance of including measures from the differing perspectives of payers, professionals and patients. This work has come a long way with the work of the Institute for Health care Improvement and their introduction of the "Triple AIM." This balance is reflected in Center for Medicare \& Medicaid Services' (CMS) national measure sets for accountable care organizations which include measures of efficiency, clinical outcomes, and patient satisfaction. ${ }^{7}$ However, these measures remain weak in truly addressing cost variations for patients from setting to setting, the impact of hidden costs for the patients and no national measures provide information as to the patient's perspective of whether they have received what they need for better health.

The movement to value-based payments or shared savings programs based on quality metrics addresses some the challenges identified with previous cost containment attempt; however, our progress toward paying for improvement is fraught with complexities and concerns. We have a long way to go to fully uncover the true cost of care in a system focused on health and not just on the provision of health care. The experience with pay for performance programs is mixed and we have yet to see the progress that it seemed to have promised. Our ability to acquire and compile big data is impressive, but is the target that we are hitting on the wrong tree? How do we learn from where we are so to propel us in the right direction?

I am concerned that we are moving away from small teams of passionate staff who are a part of the care team to large QI departments that forgo the front-line staff but, in their place, include systems analysts, software engineers, and implementation specialists. While the smaller teams were energetic and impassioned, we were unable to impact large systems because we lacked the data systems, the support from our peers, and much of the skills and 
evidence that we have today. However, those small teams were steadfast in their commitment to creating positive change from small, incremental tests of changes implemented over time. Our successes were our own and we learned a lot.

While QI departments in health systems today may have the ability to make a much larger impact in a shorter period of time and show great promise in demonstrated outcomes, have we lost some of the spirit and passion for learning and applying what we learn to our own practice? I fear that we are creating a divisive culture where data are used more as a weapon than a tool for learning and testing changes. QI teams are often formed at the corporate or institutional level and may be viewed as adversarial to direct care teams. Can the medical specialty boards help to emphasize the importance of a local focus by requiring physicians to participate in meaningful QI work? We must work to drive advancements in the field of QI without losing the critical piece of including the front-line staff and providers in the process. We must recognize the need for expertise in all areas of improvement. Allowing providers to have the time to engage in the application of QI tools and techniques is a key piece in advancing these efforts. This can be enhanced by recognizing and rewarding providers' efforts in making improvements to care by continuing to require it as part of the maintenance of certification process.

It is imperative that we balance the appeal of systemic change by mandating improvements with increasing our capacity to learn and apply our experiences with various changes to better understand the data and the story it tells. There is evidence that we can create a culture where QI activities engage providers in the changes that matter to them while also helping them meet the requirements of maintaining their certification, pay for performance and improved patient care. ${ }^{8-10} \mathrm{I}$ fear that the farther removed the QI team is from the examination room or the hospital bed, the easier it might be to see the data as numbers and not as patients. By requiring physicians to continue to learn how to apply QI techniques by testing their ideas and changes as a part of their maintenance of certification process, we can also help to appropriately balance the input that we have on QI teams and our ability to continually strive to learn so we can all improve.

Since this commentary was drafted, the COVID-19 pandemic hit, forcing overnight innovations, inflicting financial hardships for many primary care practices and furloughs of staff throughout health systems. What does this mean for QI? The transformation of care delivery systems in practices has never been more relevant. Providers are adopting virtual care, developing new diagnostic workflows, and conducting outreach to nursing homes and vulnerable populations. Family physicians are fully engaged in the changes that are happening now and are critical in helping to envision a new health care delivery model. Supporting organizations provide forums and virtual platforms, such as the American Board of Family Medicine Performance Improvement (PI) Modules and resources from Area Health Education Centers or the Institute for Health care Improvement, which can provide some structure to embolden learning while navigating the sea change that lies ahead.

Just as important, however, is the work needed to build the system of health care for after the pandemic. What can we learn from changes that we make today to inform the systems that we want in our future? How do we ensure that we swing the QI pendulum around from small, isolated groups of enthusiastic front-line care team members and large systemlevel data teams to a combination of impassioned providers in search of meaningful patient outcomes reinforced by data analytics and the supportive QI expertise? If we embrace a culture that harnesses care team members' desire for and involvement in improving their patients' experiences and outcomes to motivate change, we will find a better way for the data analysts and QI experts to guide and support the work. During this pandemic, our health care system has demonstrated that it can be dynamic and quickly make the changes that need to be made to adapt and respond to new models of care, even on the fly. Imagine what health care could look like if we continue to channel that energy into improving our care after the pandemic and with the support of all the resources that we have grown and developed within QI over that past decades.

To see this article online, please go to: http://jabfm.org/content/ 33/Supplement/S42.full.

\section{References}

1. Newton WP, Lefebvre A, Donahue KE, Bacon T, Dobson A. Infrastructure for large-scale qualityimprovement projects: early lessons from North Carolina Improving Performance in Practice. J Contin Educ Health Prof 2010;30:106-13.

2. Cykert S, Lefebvre A, Bacon T, Newton W. Meaningful use in chronic care: improved diabetes 
outcomes using a primary care extension center model. N C Med J 2016;77:378-83.

3. Halladay JR, DeWalt DA, Wise A, et al. More extensive implementation of the chronic care model is associated with better lipid control in diabetes. $\mathrm{J}$ Am Board Fam Med 2014;27:34-41.

4. Agency for Health Research and Quality's Case Studies of Leading Primary Care Practice Facilitation Programs-Program snapshot: North Carolina's support efforts. Available from: https:// pcmh.ahrq.gov/sites/default/files/attachments/ NorthCarolina_020413comp_0.pdf.

5. Mcglynn EA. Six challenges in measuring the quality of health care. Health Affairs 1997;16:7-21.

6. Berwick DM, Nolan TW, Whittington J. The Triple Aim: Care, health, and cost. Health Affairs 2008;27:759-69.
7. Program Guidance \& Specifications. Available from: https://www.cms.gov/Medicare/Medicare-Fee-forService-Payment/sharedsavingsprogram/programguidance-and-specifications.

8. Peterson LE, Blackburn B, Puffer JC, Phillips RL, Jr. Family physicians' quality interventions and performance improvement for hypertension through maintenance of certification. J Healthcare Qual 2016;38:175-86.

9. Marshall TL, Jerardi KE, Shah SS. Making MOC quality improvement participation meaningful. Pediatrics 2018;142:e20182559.

10. Stephenson CR, Wittich CM, Pacyna JE, Wynia MK, Hasan O, Tilburt JC. Primary care physicians' perceptions of practice improvement as a professional responsibility: a cross-sectional study. Med Educ Online 2018;23:1474700. 